\title{
DESEMPENHO OPERACIONAL DO CONJUNTO TRATOR- IMPLEMENTOS NAS OPERAÇÕES DE PREPARO DO SOLO
}

CORTEZ, Jorge Wilson ${ }^{1}$

FERREIRA, Bernardo José Marques²

ALVES, Aline Dantas da Silva ${ }^{2}$

MOURA, Marcelo Rubens Dias de ${ }^{2}$

NAGAHAMA, Hideo de Jesus ${ }^{3}$

\begin{abstract}
RESUMO: Os diversos sistemas de preparo do solo podem afetar o desempenho do trator agrícola devido as interações solo-máquina. O objetivo do presente trabalho foi avaliar o desempenho operacional do trator agrícola nas operações de preparo do solo com escarificador, grade em tandem, e grades leve off-set com discos de 0,56 e 0,61 m e o número de operações sobre a mesma área (uma, duas e três). O trabalho foi realizado no Campus Ciências Agrárias da UNIVASF - Petrolina (PE) com o delineamento em blocos em esquema de parcela subdividida 4x3 com 4 repetições. Foram mensurados: velocidade de deslocamento, capacidade de campo efetiva e operacional e o tempo efetivo de trabalho. A velocidade de deslocamento não foi alterada em função dos implementos. A capacidade de campo efetiva, operacional e o tempo efetivo de trabalho foram influenciados pelos implementos de preparo e o número de operações na área.
\end{abstract}

Palavras-chave: Preparo convencional. Grade. Escarificador.

\section{PERFORMANCE TRACTOR-IMPLEMENTS IN OPERATIONS OF SOIL TILLAGE}

SUMMARY: The various tillage systems can affect the performance of the tractor due to soil-machine interactions. The objective of the present work was to evaluate the operational acting of the agricultural tractor in the soil tillage operations with chisel plow, disc harrow, and two disc harrow, and the number of operations on the same area (one, two three). The work was accomplished in UNIVASF - Petrolina (PE) with the design in blocks in split plot $4 x 3$ with 4 repetitions. They were measured: forward speed, field capacity, operational and effective working time. The forward speed did not changed with the implements. The effective field capacity, operational and effective working time were influenced by tillage implements and the number of operations in the area.

Keywords: Tillage. Disc harrow. Chisel plow.

1 Eng. Agrônomo, Prof. Adjunto, Colegiado de Eng. Agronômica, Universidade Federal do Vale do São Francisco - UNIVASF. Rod. BR $407 \mathrm{Km} 12$ Lote $543 \mathrm{PSNC}$, s/n $\mathrm{n}^{\circ}$ - C1. Petrolina - PE. Email: jorge.cortez@univasf.edu.br

2 Egresso do curso de Zootecnia da UNIVASF. bernardojmf@hotmail.com alineunivasf@gmail.com mrdmoura@yahoo.com.br

3 Eng. Agrônomo, Chefe da Seção de Apoio as Atividades de Campo da UNIVASF. Email: hideo.nagahama@univasf.edu.br 


\section{INTRODUÇÃO}

As máquinas agrícolas devem estar adequadas, e ter capacidade satisfatória para executar as atividades de uma propriedade dentro dos períodos planejados (SILVEIRA et al., 2006). Para conseguir executar as atividades dentro de certos períodos deve-se estudar as capacidades, de campo, teórica, efetiva e operacional de modo a minimizar os erros quando da realização das operações a campo.

Dentre as operações no sistema de cultivo, o sistema de preparo do solo convencional, ou periódico, é utilizado em larga escala no Brasil, e alguns implementos apresentam capacidade operacional melhor do que outros. As máquinas para mobilização periódica do solo são aquelas destinadas a promover alterações na estrutura do solo, visando propiciar condições favoráveis a germinação das sementes (GÂMERO; LANÇAS, 1996). As grades, máquinas de preparo periódico do solo, apresentam pequeno poder de penetração no solo uma vez que, possui seus órgãos ativos fixados em um mesmo eixo (CARVALHO FILHO et al., 2007a). Porém, devido a sua maior largura de trabalho apresenta capacidade operacional elevada.

As grades por apresentarem capacidade de campo mais elevada do que outros implementos são utilizadas em maior escala no sistema de preparo periódico do solo. Verificaram que dentre as operações de aração, gradagem, cultivo e semeadura, a grade apresentou a maior capacidade de campo efetiva, $0,86 \mathrm{ha} \mathrm{h}^{-1}$, com velocidade média de $5,32 \mathrm{~km} \mathrm{~h}^{-1}$ (SILVEIRA et al., 2006).

Os implementos de preparo do solo incorporam resíduos da superfície, deixando o solo exposto ao intemperismo, mas busca-se cada vez menos a incorporação de resíduos, pois a palha sobre o solo protege das intempéries. Verificaram que quanto à incorporação de resíduos vegetais da superfície do solo, o arado de aivecas $(96,4 \%)$ foi > arado de discos $(87,8 \%)>$ grade aradora $(70,9 \%)>$ enxada rotativa $(58,8 \%)>$ escarificador $(28,4 \%)$ (CARVALHO FILHO et al., 2007b).

Fernandes e Gamero (2010) ao estudar o desempenho operacional do preparo reduzido e convencional verificaram para a capacidade de campo teórica, que a grade leve apresentou $1,28 \mathrm{ha} \mathrm{h}^{-1}$, enquanto que o escarificador foi de $0,80 \mathrm{ha} \mathrm{h}^{-1}$, sendo as velocidades de 5,01 e $2,87 \mathrm{~km} \mathrm{~h}^{-1}$, respectivamente.

O objetivo do presente trabalho foi avaliar o desempenho operacional do conjunto trator-implementos nas operações de preparo do solo e o número de operações sobre a mesma área. 


\section{MATERIAL E MÉTODOS}

O trabalho foi instalado no Campus de Ciências Agrárias da Universidade Federal do Vale do São Francisco (UNIVASF) em Petrolina - PE. A área experimental está localizada em latitude 09¹9'16" sul e longitude 40³3'43" oeste, a uma altitude de $373 \mathrm{~m}$. Segundo a classificação de Köppen, o clima da região é tropical semiárido, tipo BSwh, caracterizado pela escassez e irregularidade das precipitações com chuvas no verão e forte evaporação em consequiência das altas temperaturas. O solo foi classificado como Argissolo Amarelo, textura arenosa (EMBRAPA, 2006). A área de instalação do experimento possui sistema linear de irrigação, visando a manutenção da umidade do solo no momento do preparo e durante a coleta de dados.

Foi utilizado o delineamento em blocos ao acaso com parcelas subdivididas com quatro repetições. Os quatro tratamentos de preparo do solo, aplicados nas parcelas principais, foram: preparo com escarificador, grade leve em "tandem", grade leve "off-set" com discos de 0,56 m (22") de diâmetro e grade leve "off-set" com discos de 0,61 m (24") de diâmetro. Nas subparcelas, foram aplicadas uma, duas e três operações de mobilização do solo, com cada equipamento. Cada parcela experimental ocupou área de 20x10 m (200 m²). No sentido longitudinal entre as parcelas, foi reservado um espaço de $15 \mathrm{~m}$, destinado a realização de manobras, tráfego de máquinas e estabilização dos conjuntos de implementos.

Os equipamentos utilizados para o preparo do solo foram: (a) Grade leve em "tandem" com sete discos em cada uma das quatro seções, num total de 14 discos recortados na dianteira e 14 lisos na traseira, com diâmetro de 0,51 m (20”), distância entre discos de 0,19 $\mathrm{m}$, massa de $528 \mathrm{~kg}$ e profundidade de trabalho de $0,10 \mathrm{~m}$; (b) Grade leve "off-set" com oito discos em cada uma das duas seções, num total de 16 discos recortados com 0,56 m de diâmetro (22"), distância entre discos de $0,23 \mathrm{~m}$, massa de aproximadamente $1.000 \mathrm{~kg}$, e profundidade de trabalho de 0,15 m; (c) Grade leve "off-set" com sete discos em cada uma das duas seções, no total de 14 discos recortados com diâmetro de 0,61 m (24"), distância entre discos de $0,23 \mathrm{~m}$, massa de $1.094 \mathrm{~kg}$ e profundidade de trabalho $0,18 \mathrm{~m}$ e (d) Escarificador com três hastes espaçadas em $0,34 \mathrm{~m}$, ponteira estreita de $0,05 \mathrm{~m}$, e massa de $295 \mathrm{~kg}$ e profundidade de trabalho $0,35 \mathrm{~m}$.

Como fonte de potência para tracionar os equipamentos de preparo do solo foi utilizado um trator modelo 785 TDA (tração dianteira auxiliar), com 55,20 kW (75 cv) de potência com pneus dianteiros 12.4-24 R1 e traseiros 18.4 - 30 R1, sendo utilizada a rotação de trabalho no motor de 2.040 RPM (rotações por minuto). 
Para calcular a velocidade de deslocamento utilizou-se cronometro digital, com precisão de $0,1 \mathrm{~s}$, acionado no momento em que o trator entrava na área e desligado ao final da parcela quando o trator saia da mesma. A largura efetiva de trabalho dos equipamentos foi medida, utilizando-se trena, diretamente sobre as passadas dos equipamentos nas parcelas e suas médias foram: escarificador $(1,30 \mathrm{~m})$, grade em tandem $(2,50 \mathrm{~m})$, grade leve off-set com discos de 0,56 m (2,0 m) e grade leve off-set com disco de 0,61 m ( 1,9 m).

A capacidade de campo efetiva (Equação 1), que é a quantidade de hectares trabalhados por hora pelo conjunto, foi calculada pela velocidade real do trator e largura real do implemento.

$$
\mathrm{CcE}=\frac{\mathrm{Lm} * \mathrm{~V}}{10}
$$

em que,

CcE: capacidade de campo efetiva $\left(\right.$ ha $\left.^{-1}\right)$,

$\mathrm{V}$ : velocidade de deslocamento $\left(\mathrm{km} \mathrm{h}^{-1}\right)$,

Lm: largura de trabalho (m), e

10: fator de conversão para $\left(h a \mathrm{~h}^{-1}\right)$.

A capacidade de campo operacional (Equação 2) foi determinada com base na largura real de cada implemento e da velocidade real de deslocamento do conjunto, multiplicando-se por um fator de eficiência.

$$
\mathrm{CcO}=\frac{\mathrm{Lm} * \mathrm{~V}}{10} * E f
$$

em que,

CcO: capacidade de campo operacional $\left(\mathrm{ha}^{-1}\right)$;

$\mathrm{V}$ : velocidade de deslocamento $\left(\mathrm{km} \mathrm{h}^{-1}\right)$,

Lm: largura de trabalho (m), e

Ef: eficiência (80\%), pois segundo Balastreire (2005) variam de 70 a $90 \%$.

O tempo efetivo de trabalho foi calculado pelo inverso da capacidade de campo efetiva.

Os dados obtidos foram tabelados e submetidos à análise de variância e quando o valor do teste $\mathrm{F}$ (Fisher) foi significativo a $5 \%$ de probabilidade foi realizado o teste de Tukey para a comparação de médias (PIMENTEL GOMES, 1987). 


\section{RESULTADOS E DISCUSSÃO}

Os coeficientes de variação (CV) apresentados na Tabela 1 podem ser classificados como baixos $(<12 \%)$ ou altos $(>24 \%)$ segundo Warrick \& Nielsen (1980), para o CV das subparcelas foram baixos $(<12 \%)$, e médios (de 12 a 24\%) para o CV da parcela, mas percebe-se que o $\mathrm{CV}$ da parcela estão bem próximos de $12 \%$. Verifica-se que as interações entre equipamentos e operações não foram significativas no teste de Fisher $(\mathrm{F})$, por isso apresentados os resultados pela média, e que a capacidade de campo efetiva (CcE), capacidade de campo operacional $(\mathrm{CcO})$ e tempo efetivo de trabalho (Tef) foram significativos nos fatores equipamentos e número de operações.

Tabela 1. Síntese de análise de variância para os componentes operacionais equipamentos e operações e as variáveis: velocidade de deslocamento (V), capacidade de campo efetiva (CcE), capacidade de campo operacional $(\mathrm{CcO})$ e tempo efetivo de trabalho (Tef).

\begin{tabular}{lcccc}
\hline TESTE DE F & $\mathrm{V}$ & $\mathrm{CcE}$ & $\mathrm{CcO}$ & Tef \\
\hline Equipamentos (E) & $3,1^{\mathrm{ns}}$ & $61,6^{* *}$ & $73,94^{* *}$ & $67,1^{* *}$ \\
Operações (O) & $1,6^{\mathrm{ns}}$ & $6,6^{*}$ & $8,69^{* *}$ & $7,6^{* *}$ \\
E x O & $1,3^{\mathrm{ns}}$ & $1,4^{\mathrm{ns}}$ & $1,78^{\mathrm{ns}}$ & $0,68^{\mathrm{ns}}$ \\
\hline C.V. Parcela & 11,1 & 12,6 & 12,1 & 12,3 \\
\hline C.V. Subparcela & 6,5 & 8,6 & 7,6 & 8,0 \\
\hline${ }^{{ }^{\mathrm{n}}}$ : não significativo (P>0,05); *: significativo $(\mathrm{P} \leq 0,05) ; * *$ significativo $(\mathrm{P} \leq 0,01) . \mathrm{CV}$ : coeficiente de variação
\end{tabular}
$(\%)$.

A velocidade de deslocamento não foi significativa $(\mathrm{P}>0,05)$ para os implementos e operações (Tabela 2). Observa-se que os implementos de preparo do solo apresentaram velocidade média de $5,46 \mathrm{~km} \mathrm{~h}^{-1}$. Esperava-se variação na velocidade em virtude de se trabalhar com implementos de diferentes larguras de trabalho e profundidades. No entanto, o implemento com menor largura, escarificador $(1,20 \mathrm{~m})$, trabalhou na maior profundidade (0,35 m), comparado com as grades, em tandem, off-set com discos de 0,56 m de diâmetro e off-set com discos de $0,61 \mathrm{~m}$ de diâmetro que trabalharam em 0,10, 0,15 e 0,18 m, respectivamente. Provavelmente os diferentes equipamentos exigiram do trator, potência na barra, muito próxima, o que resultou em velocidades semelhantes na análise. Salvador et al., (2008) verificaram que houve variação de velocidade entre os implementos de preparo periódico do solo, em virtude de terem sido projetado para diferentes condições, discordando dos resultados deste trabalho. Fernandes \& Gamero (2010) verificaram velocidades de 5,01 e 2,87 $\mathrm{km} \mathrm{h}^{-1}$ para as operações com grade leve e escarificador, respectivamente, resultado similar para a grade, mas diferente do escarificador, devido a largura de trabalho dos implementos. 
Para as capacidades de campo (Tabela 2) verifica-se efeito similar para os implementos $(\mathrm{P}<0,01)$, uma vez que a grade em tandem apresentou a maior capacidade, enquanto que o escarificador a menor, isto está relacionado com a largura de trabalho de cada implemento, sendo que a grade em tandem apresenta a maior largura e o escarificador a menor largura. Silveira et al, (2006) verificaram para o preparo com grade que a capacidade de campo efetiva foi de $0,86 \mathrm{ha}^{-1}$, valor este, inferior ao deste trabalho, mesmo se comparado com a capacidade de campo operacional, provavelmente devido a utilização de velocidades de trabalho diferentes.

Tabela 2. Síntese de análise de variância para os componentes operacionais: velocidade de deslocamento $(\mathrm{V})$, capacidade de campo efetiva $(\mathrm{CcE})$, capacidade de campo operacional $(\mathrm{CcO})$ e tempo efetivo de trabalho (Tef).

\begin{tabular}{lccrr}
\hline Fatores & $\mathrm{V}\left(\mathrm{km} \mathrm{h}^{-1}\right)$ & $\mathrm{CcE}\left(\mathrm{ha} \mathrm{h}^{-1}\right)$ & $\mathrm{CcO}\left(\mathrm{ha} \mathrm{h}^{-1}\right)$ & $\mathrm{Tef}_{\left(\mathrm{h} \mathrm{ha}^{-1}\right)}$ \\
\hline EQUIPAMENTOS & \multicolumn{4}{c}{} \\
\hline Escarificador & 5,45 & $0,72 \mathrm{c}$ & $0,56 \mathrm{~d}$ & $1,41 \mathrm{a}$ \\
Grade em tandem & 5,76 & $1,43 \mathrm{a}$ & $1,16 \mathrm{a}$ & $0,70 \mathrm{~d}$ \\
Grade leve off-set $^{(1)}$ & 5,60 & $1,10 \mathrm{~b}$ & $0,89 \mathrm{~b}$ & $0,92 \mathrm{c}$ \\
Grade leve off-set $^{(2)}$ & 5,04 & $0,93 \mathrm{~b}$ & $0,75 \mathrm{c}$ & $1,08 \mathrm{~b}$ \\
\hline OPERAÇÕES & 5,36 & $1,00 \mathrm{~b}$ & $0,80 \mathrm{~b}$ & $1,08 \mathrm{a}$ \\
\hline Uma & 5,43 & $1,03 \mathrm{~b}$ & $0,84 \mathrm{ab}$ & $1,01 \mathrm{ab}$ \\
Duas & 5,59 & $1,11 \mathrm{a}$ & $0,89 \mathrm{a}$ & $0,97 \mathrm{~b}$ \\
Três & 5
\end{tabular}

Médias seguidas de mesma letra na coluna não diferem pelo Teste de Tukey. ${ }^{(1)}$ Grade leve off-set com discos de $22 ”(0,56 \mathrm{~m}) \mathrm{e}$; ${ }^{(2)}$ Grade leve off-set com discos de $24 ”(0,61 \mathrm{~m})$.

A capacidade de campo efetiva e operacional (Tabela 2) em função do número de operações foi significativa $(\mathrm{P}<0,01)$, de tal forma que, o aumento no número de operações apresentou maior capacidade (com três operações na área), que pode estar relacionado com a menor resistência do solo, em virtude de ter sido preparado por duas vezes.

O tempo efetivo de trabalho foi significativo $(\mathrm{P}<0,01)$ para implementos e operações sendo o maior tempo gasto com a operação de escarificação, e o menor com a grade em tandem, este fato está relacionado a largura de trabalho. O maior tempo efetivo de trabalho diferiu com uma de três operações, sendo que com uma operação obteve-se o maior tempo de trabalho, que pode estar relacionado a maior resistência do solo no momento do primeiro preparo. Verifica-se que a variação nas capacidades de campo e no tempo efetivo de trabalho está relacionada somente a largura de trabalho, pois a velocidade não foi significativa.

O escarificador é considerado um equipamento de preparo periódico conservacionista por manter maior cobertura do solo (CARVALHO FILHO et al., 2007a). A baixa capacidade operacional apresentada neste trabalho, se deve a menor largura de trabalho do implemento, 
mas Levien et al., (2003) demonstraram que com o escarificador (preparo reduzido) é possível obter maior desempenho operacional.

\section{CONCLUSÃO}

A velocidade de deslocamento foi constante mesmo com equipamentos de diferentes larguras de trabalho.

A capacidade de campo efetiva, operacional e o tempo efetivo de trabalho foram influenciados pelos diferentes equipamentos de preparo e o número de operações na área.

\section{AGRADECIMENTOS}

A Universidade Federal do Vale do São Francisco - UNIVASF, pela concessão da bolsa de estudo do ao segundo, terceiro e quarto autor.

\section{REFERÊNCIAS}

BALASTREIRE, L.A. Máquinas Agrícolas. Piracicaba: Balastreire, 2005. 307p.

CARVALHO FILHO, A.; CENTURION, J.F.; SILVA, R.P.; FURLANI, C.E.A.; CARVALHO, L.C.C. Métodos de preparo do solo: alterações na rugosidade do solo. Engenharia Agrícola, Jaboticabal, v. 27, n. 1, p. 229-237, 2007 a.

CARVALHO FILHO, et al.. Agregação de um latossolo vermelho submetido a cinco sistemas de preparo do solo em Uberaba - MG. Engenharia Agrícola, Jaboticabal, v. 27, n. 1, p. 317 $325,2007 b$.

EMPRESA BRASILEIRA DE PESQUISA AGROPECUÁRIA - EMBRAPA. Sistema Brasileiro de Classificação de Solos. Brasília: Centro Nacional de Pesquisas de Solos, 2006. 370p.

FERNANDES, J.C.; GAMERO, C.A. Avaliação do desempenho das máquinas agrícolas na implantação da cultura do girassol. Energia na Agricultura, Botucatu, vol. 25, n.2, p.74-87, 2010.

LEVIEN, R.; GAMERO, C. A.; FURLANI, E. A. Manejo convencional e reduzido em solo argiloso em diferentes condições de cobertura de inverno. Engenharia Agrícola, Jaboticabal, v. 23, n. 2, p. 277- 289, 2003.

GÂMERO, C.A.; LANÇAS, K.P. Ensaio \& certificação das máquinas de mobilização periódica do solo. In: MIALHE, L.G. Máquinas agrícolas: ensaio e certificação. Piracicaba: Fundação de Estudos Agrários Luiz de Queiroz, 1996. Cap. 9, p. 463-514. 
PIMENTEL GOMES, F. A estatística moderna na agropecuária. Piracicaba: Associação Brasileira para Pesquisa da Potassa e do Fosfato, 1987. 162p.

SALVADOR, N.; BENEZ, S.H.; MION, R.L.; VILIOTTI, C.A. Demanda energética em diferentes sistemas de preparo periódico do solo antes e depois da subsolagem. Ciência Agronômica, Fortaleza, v. 39, n. 3, p. 378-383, 2008.

SILVEIRA, G.M. da; YANAI, K.; KURACHI, S.A. H. Determinação da eficiência de campo de conjuntos de máquinas convencionais de preparo do solo, semeadura e cultivo. Revista Brasileira de Engenharia Agrícola e Ambiental, Campina Grande, v. 10, n. 1, p. 220-224, 2006.

WARRICK, A.W.; NIELSEN, D.R. Spatial variability of soil physical properties in the field. New York, Academic Press, 1980. 